Jakob Norberg

\title{
Der Text als Phrase: Schillerfeier und geflügelte Worte
}

\section{Klage}

Am 19. Mai 2007 findet die Premiere von Peter Steins Wallenstein-Inszenierung in Berlin statt, mit dem „„̈sterreichische[n] Großschauspieler“ Klaus Maria Brandauer als Hauptdarsteller. ${ }^{1}$ Die Aufführung umfasst zehn oder elf Stunden; die Einschätzungen variieren. Bereits am nächsten Tag besucht Rainald Goetz die Darbietung. Anschließend publiziert er einen „Kurzreport“ auf seinem Blog Klage. ${ }^{2}$ Er kehrt nicht ermüdet oder enttäuscht von der Aufführung zurück, er ist entsetzt. Alles an diesem Event sei scheußlich. Die Inszenierung ist „zäh“, „lang, doof, platt, dahergeknattert“ und Brandauer schlicht „der scheußlichste Mensch auf Erden.“3 Schuld an der Katastrophe seien aber nicht nur Brandauer oder Stein, sondern auch Schiller: „Wallenstein ist schlecht, schlechtes Theater vom Text her" ${ }^{\star 4}$ und Schiller ,[d] as zweitscheußlichste“ ${ }^{\text {" }}$ nach Brandauer.

So weit der Kurzreport unmittelbar nach der Aufführung. Goetz bleibt aber bei dem Thema und stellt auch am nächsten Tag drei Absätze ins Netz. Jetzt geht es nicht um Brandauer, sondern um das grundlegende Problem, also um Schiller. Warum ist Wallenstein so schlecht?

Als Stückeschreiber ist Schiller der Mann der Phrase. Das macht seinen Erfolg, die sprachliche Vereinfachung, der simple Spruch, wo jeder nickt und sagen kann: das stimmt, das habe ich mir auch schon oft gedacht. Es stimmt nur genau deshalb ja auch genau nicht. Und der lange traurige Triumphzug dieser biederen Kalauer und verbal brutal abgestumpften Lebensweisheiten in Schillers Stücken durch die Spießerkultur des 19. Jahrhunderts ins moderne aggressive Hochspießertum im Nationalsozialismus, wo Schiller ultimativ abgefeiert wurde, liegt in Schiller selbst begründet, in der geistigen Kleinheit seiner Sprüchewelt. ${ }^{6}$

„Phrase“ ist hier Goetz’ Bezeichnung für vermeintlich allgemeingültige Aussagen oder Urteile, die breite Zustimmung nur durch Vereinfachung sichern. ${ }^{7}$ Der zusammenfassende Spruch meidet oder negiert Komplexität, wird deswegen von allen verstanden und von vielen bejaht (der Spießer funktioniert in diesem Kontext wohl als Bezeichnung einer Figur, die ,soziale und kulturelle Unübersichtlichkeit“" nicht bewältigen will oder kann ${ }^{8}$ ). Das ist rhetorisch gesehen genau beschrieben: Die „delectatio-Wirkung“ von Schillers Sprüchen oder Sentenzen, so Gert Ueding in seiner Studie zu Schillers Rhetorik, beruht

1 Spiegel-Online-Rezension von Jenny Hoch, zitiert in Esther Slevogt: „Überwältigungsästhetik im Breitwandformat", www.nachtkritik.de, unter http://www.nachtkritik.de/index.php?option=com content\&view=article $\& \mathrm{id}=191 \% 3$ Awallenstein-peter-steins-schiller-marathon-in-der-neukoellner-kindlhalle\&catid=50\&Item $\mathrm{id}=100077$

2 Goetz (2008), S. 137.

Ebd., S. 138.

Ebd., S. 139.

Ebd., S. 138.

Ebd., S. 140 - 141

Phrase gilt als Begriff für feste und deswegen reproduzierbare, aber tendenziell inhaltslose Formulierungen: „Seit Mitte des 18. Jh. wird 'Ph.' ensprechend frz. phrases ('leere Worte') gewöhnlich abwertend gebraucht im Sinne von ,inhaltlose Aussage, leeres Geschwätz, Leerformel' " "Wenzelburger/Kaiser (2007), S. 585.

$8 \quad$ Vogl/Kluge (2009), S. 70. 
auf der Freude des Zuhörers darüber, dass jemand in einem ,allgemeinen Satz“ seine eigene Ansicht trifft. ${ }^{9}$

Goetz ist nicht der Einzige, der Schillers Sentenzen als Phrasen bezeichnet und kritisiert hat. Die Behauptung, Schiller sei der Mann der Phrase, weist eine 200-jährige Geschichte auf; sie tritt unmittelbar nach seinem Tod in Erscheinung. Ich vermeide es hier, von einer Tradition zu sprechen, denn man kann sich fragen, ob eine Tradierung tatsächlich stattgefunden hat. Aber viele haben Schiller gelesen, seine Rezeption verfolgt und sind zu vergleichbaren Urteilen gekommen, über die Verfasstheit seiner Sprache, seine Fähigkeit, Sprüche zu prägen, und die damit zusammenhängende Popularität, die in der deutschen Literaturgeschichte fast einzigartig ist. Einige Beispiele dieser hartnäckigen Kritik der Phrase möchte ich im Folgenden vorlegen und dabei die Frage stellen, was eigentlich kritisiert wird. ${ }^{10}$

\section{Phrasenkritik}

In der 1811 publizierten „,scherzhafte[n] Abhandlung“ „Der Philister vor, in und nach der Geschichte" behauptet Clemens Brentano, Schiller sei deswegen unter kulturfeindlichen Philistern ${ }^{11}$ so beliebt, weil sich seine Werke leicht in unabhängige Sentenzen oder Stückchen auflösen lassen:

Sie [die Philister] traben, solange es geht, mit der neusten Ästhetik mit, und würgen das Zeug aus Hoffart ungekaut hinunter; je gröber sich ihr Autor brocken läßt, je heftiger würgt sie der Bissen, und je größer ist der Genuß, drum lieben sie den herrlichen Schiller vorzüglich, weil sie seine sentenziöse reflektierende Diktion in lauter Stammbuchstückchen zerknicken und verschlingen können. ${ }^{12}$

Philister lieben Schiller, weil seine Texte knappe, abgesetzte Sätze beinhalten, die sich herausbrechen lassen. Das typische Schillerwerk ist voll von Sentenzen, also kurzen Formulierungen ,allgemeine[r] Gedanken inmitten einer Dichtung ${ }^{\text {“13 }}$, und leistet deswegen einem eher „kulinarischen“ Zugang keinen Widerstand. ${ }^{14}$ Es lässt sich etwas von Schiller in den Mund nehmen - die sentenziöse Form kann metaphorisch mit einem oralen, also vulgären Genuss verbunden werden. Beim lesenden Philister geht es um Verschlingen, Schlürfen und Verdauung, wie idealistisch die angeeignete Literatur auch sei: „So gehen sie [die Philister] in den Kosegarten [Ludwig Gotthard Kosegarten, deutscher Dichter] spazieren und sprechen mit ekelsüßer Miene: ,Horch, wie orgelt und brauset die Äolsharfe der Schöpfung'; doch eigentlich orgelt und brauset ihr Magen nur". ${ }^{15}$ Bei Brentano wird der hohe Schiller-Ton konsequent von derben Bildern unterlaufen.

9 Ueding (1971), S. 182. Ueding stützt sich auf Aristoteles' Rhetorik.

10 Für anregende Hinweise danke ich Natalie Binczek und Georg Stanitzek. Das Projekt wurde durch die Volkswagen-Stiftung gefördert.

11 Über den Philister als Sozialfigur der deutschen Literatur- und Kulturgeschichte siehe Bunia, Dembeck/ Stanitzek (2011)

12 Brentano (1963)

13 Niemeyer (1934), S. 27

14 Die kulinarische Metaphorik kommt z. B auch bei Gottfried Benn vor: Wir befinden uns im Zeitalter der Aphorismen und Anthologien, [...] man kann auch sagen im Zeitalter der Erleichterung der schweren Dinge. Mundgerecht soll alles gemacht werden, keiner soll mehr an einer selbstbestellten und selbstbereiteten Hauptnahrung herumkauen müssen [...], also: kleine Bissen, vorgekaut, weichgekocht“. Gottfried Benn: „Anthologien. Zum ,Tag des Buches‘ am 27. November 1954“, in: Benn (2001), S. 158.

15 Ebd. 
Eine weitere Station der Schillerkritik: Karl Kraus' Die Fackel. Anlässlich der Schillerfeiern im Jahr 1909 weist Kraus in dem Artikel „Schrecken der Unsterblichkeit“ auf die Verbindung zwischen der enthusiastischen Schillerverehrung und seinen „Schatzkammer[n] der Banalität“ hin, d.h. auf Schillertexte, die in Phrasen zerfallen und leicht geplündert werden: ${ }^{16}$

,Wie sagt doch Schiller... Alle jene, die so anfangen, wenn sie zur Quelle ihrer Banalität führen wollen, müssen erst vom Schauplatz des deutschen Geisteslebens weggeputzt werden, ehe wir uns überhaupt wieder in ein Verhältnis zu Schiller setzen lassen. Was sie an ihm anbetungswürdig finden, sind Ideen, die als Phrasen gestorben sind, wenn sie nicht als Phrasen geboren wurden. ${ }^{17}$

Kraus schwankt in seiner Beurteilung, scheint Schiller nicht verwerfen zu wollen, findet es aber schwer zu behaupten, Schiller sei lesenswert. Die von Schillerverehrern immer wieder zitierten Phrasen bilden eine Schnittstelle zwischen der angeblichen Genialität und Größe des gefeierten Autors und der Idiotie seiner zahlreichen Fans. Das führt bei Kraus zu einer Art kognitiver Dissonanz; etwas muss nachgeben. Das aufdringliche Feiern von Schiller liegt auch für Kraus letztendlich in Schiller selber begründet, in den Vorräten an Phrasen, die seine Werke anbieten oder ausmachen. Bei Kraus fällt allerdings die Metaphorik teilweise weniger abwertend aus als bei Brentano: Es geht um Schätze, nicht um Bissen, ${ }^{18}$ wobei Kraus an einer Stelle die Schillerverehrer hervorkriechende „Leichenwürmer der Unsterblichkeit" nennt. ${ }^{19}$ Die Schillerrezeption bedeutet die Zersetzung seiner Werke durchs Zitieren, was als Verwesungsprozess vorgestellt werden kann.

Noch eine Station der Schillerkritik und eine weitere in ihrem Zusammenhang einschlägige Metaphorik finden sich bei Theodor Fontane:

Wann wird die Welt einsehen, dass diese Schillerphrasen eine schillernde Seifenblase sind? Unerträglich sind mir in all diesen Stücken die Biederkeitsschwätzer, die immer das Maul voll Tugend, Treue und Freundschaft haben und persönlich nichts leisten, während sie beständig alles fordern. ${ }^{20}$

Dies notiert Fontane 1883 in seinem Tagebuch. Hier sind Schillerzitate keine Stückchen, Brocken oder bürgerlicher „Ofenschmuck“21, sondern substanzlose Täuschung; als Phrasen sind sie vor allem „leere Worte“, „leeres Geschwätz“.22 Phrasen als Blasen: Das Bild bezieht sich weniger auf die unbestreitbare Festigkeit und Reproduzierbarkeit der Schiller zu entnehmenden Wendungen als vielmehr auf die moralische Fragwürdigkeit ihres Einsatzes. Es geht Fontane nicht - wie Kraus - um die Banalität der Phrasen, sondern um mangelnde Tätigkeit, die dann schon an Schillers Dramenfiguren zu bemerken ist. Fontane artikuliert, könnte man meinen, die eher konservative oder männliche Kritik der Phrase: Leute reden leichtfertig über Ideale (Tugend, Treue, Freundschaft), unternehmen aber nichts. Phrasen treten dann auf, wenn Aussagen nicht mit Taten übereinstimmen, aus der einen oder anderen Form von Unfähigkeit: Faulheit, Feigheit, Verwöhntheit.

Bei Brentano, Kraus und Fontane fehlt aber ein Element, das für die Semantik nach 1945 von großer Bedeutung ist: Goetz verknüpft Schillers Sprüche oder Phrasen und die spießbürgerliche Schillerverehrung mit dem Nationalsozialismus als der aggressivsten

16 Kraus (1909), S. 24.

17 Ebd., S. 27.

18 Die von Kraus verwendete Metaphorik wurde auch von Schillerverehrern gebraucht: Extrahierte Lebensweisheiten wurden als ,unvergängliche Schätze“ gepriesen. Siehe Darsow (2000), S. 236.

19 Ebd., S. 23.

20 Zitiert in Carbe (2005), S. 67.

21 Kraus (1909), S. 27.

22 Zur Bedeutung des pejorativen Begriffs siehe Fußnote 7. 
Form des deutschen Spießertums. ${ }^{23}$ : Die Assoziation muss anscheinend nicht weiter begründet werden. Eine vergleichbare Konstellation von Sprachkritik, Kleinbürgerporträt und Faschismusvorwurf gibt es bei Theodor W. Adorno in Minima Moralia:

53 Schwabenstreiche. - Der sprachliche Habitus Schillers gemahnt an den jungen Mann, der von unten kommt und, befangen, in guter Gesellschaft zu schreien anfängt, um sich vernehmlich zu machen: power und patzig. Die deutsche Tirade und Sentenz ist den Franzosen nachgeahmt, aber am Stammtisch eingeübt. In den unendlichen und unerbittlichen Forderungen spielt der Kleinbürger sich auf, der mit der Macht sich identifiziert, die er nicht hat, und durch Arroganz sie überbietet bis in den absoluten Geist und das absolute Grauen hinein. ${ }^{24}$

Adornos Argumentation ist hier nicht vollkommen durchsichtig. Der Text leidet an zu vielen und zu schnellen Szenenwechseln. Im ersten Satz sind wir bei dem nervös-pompösen jungen Mann, einem Fremden in guter Gesellschaft, im zweiten dann plötzlich am Stammtisch, vielleicht beim ,ritualisierten Austausch mit Gleichgesinnten bei Wein und Bier“" ${ }^{25}$ Und im dritten Satz stehen wir schließlich vor dem Kleinbürger, dessen Hilflosigkeit und Geltungsbedürftigkeit sich in verschiedene Formen der grausamsten Arroganz verwandeln. Ähnlich wie bei Fontane geht es auch hier um als Phrasen wahrnehmbare übertriebene Ansprüche, aber die skizzierte Psychologie ist komplexer. Leute, die zu viel und zu laut reden, die schwätzen, schreien und sich aufspielen, sind bei Adorno nicht einfach handlungsunfähig, sondern durch Machtlosigkeit gekränkte Subjekte. Am Ende erweisen gerade sie sich als gefährlich.

Immerhin ist das schon genannte Assoziationsbündel - Sentenzen, Kleinbürger, Faschisten - bei Adorno vorhanden; eine ähnliche Zusammenstellung gibt es bei Goetz. Vermeintlich universell gültige Aussagen und einprägsame Deklamationen bei Schiller appellieren an Personen, die nur reduktiv - schließlich nur aggressiv - mit der Welt umgehen können. Die Konstellation unterstellt, dass Sprüche breite Zustimmung oder sozialen Erfolg gerade durch ihre „brutale“ oder „,arrogante“ Tilgung von Komplexität oder Heterogenität erreichen.

\section{Schillerrezeption}

Goetz, Brentano, Kraus und Adorno entfalten zum Teil unterschiedliche Argumente, stimmen aber hierin überein: Schillers aus einprägsamen Sentenzen oder Phrasen gemachte Unsterblichkeit gilt als Indiz für seine Schwäche, seine Rezeption als deren kulturgeschichtliche Enthüllung. Auch die Germanistik behandelt Schillers problematische Beliebtheit, versucht aber, Schiller selber weitgehend zu schonen. In diesem Kontext wird statt von Phrasenhaftigkeit von Prägnanz gesprochen und die Popularität unter SpieBern als andauerndes Missverständnis beklagt. Schiller selber ist also nicht schuld an der Fragmentierung seiner Werke. Wenn die sprachliche Verfasstheit dieser Werke eine solche Fragmentierung erlaubt, dann nur, weil er ein „Meister der Sentenz klassischer Prägung“ war, keine an sich schlechte Fähigkeit. ${ }^{26}$ Dies lautet dann so:

\footnotetext{
23 Der Soziologe Michael Mann bestreitet die These, die NSDAP sei eine Partei der „Spießbürger“ gewesen. Siehe Mann (2004).

24 Adorno (2004), S. 99.

25 Bullinger (2013), S. 7.

26 Ueding (1971), S. 183.
} 
Kennzeichnend für die Schillerrezeption ist offensichtlich ein Verfahren, das die Texte in ihre Bestandteile auflöst, fragmentiert und einzelne Verse in außerliterarischen Bereichen zitiert und benutzt. [...] Zitiert werden vor allem die [. . . Sentenzen aus Dramen und Gedichten, die sich aufgrund ihrer lautlich-rhythmischen Prägnanz und relativen Kontextunabhängigkeit dem Gedächtnis einprägen und dann in den unterschiedlichsten Texten und neuen Kontexten aktualisiert werden können. ${ }^{27}$

Oder, ein bisschen defensiver:

Im Laufe des 19. Jahrhunderts bildete sich eine ungeheure Popularität Schillers aus, die aber nicht auf der Kenntnis und der produktiven Rezeption seiner Werke beruhte, sondern hauptsächlich in einer Funktionalisierung einzelner Zitate und Sentenzen aus seinen Werken bestand, die in einem häufig ganz vordergründigen Sinne aktualisiert und auf konkrete Lebenssituationen bezogen wurden. $[\ldots]$

Die populäre Rezeption Schillers, die gleich zu Beginn des 19. Jahrhunderts einsetzt, ist durch eine Trivialisierung und Instrumentalisierung des Dichters und seines Werkes gekennzeichnet. Ja, nicht das Werk wurde eigentlich rezipiert, sondern Sentenzen, die aus dem Werkzusammenhang gerissen wurden. ${ }^{28}$

Die Sentenziösität von Schillers Sprache wird nicht geleugnet. Aber die systematische Zersetzung und Verstreuung seiner Dramen und Gedichte ist dennoch Ergebnis eines von außen applizierten Verfahrens, das als Benutzung, Funktionalisierung und Instrumentalisierung bezeichnet wird. Was als „fragmentierend-sentenziöse Selbstbedienung der Leser bei den Texten“" ${ }^{\text {"29 }}$ anfängt, verselbständigt sich auch schnell, indem die meisten, die Schiller enthusiastisch nachsprechen, Schiller kaum gelesen haben oder nachlesen. Am Ende gilt Schiller nur als „Verfasser der Sentenzen“ und seine eigentlichen Texte erscheinen bestenfalls als „Beiwerk zu seinen Kernsprüchen und Lebensweisheiten“ “ ${ }^{30}$ Schiller gehört dann fast zu einem Kreis von Autoren (La Rochefoucauld, Lao Tzu) „known almost exclusively through their proclamations ${ }^{\text {“31 }}$. Und da das eigentliche Werk - sagen wir: seine ästhetische Integrität, seine gedankliche Komplexität und Tiefe usw. - weggedrängt wird, öffnet sich bei Schiller wie bei kaum einem anderen Autor eine Kluft ,zwischen Popularität und Verständnis"“. ${ }^{32}$

„Als Stückeschreiber“, schreibt Rainald Goetz, ist Schiller „,der Mann der Phrase“, 33 aber gerade als Mann der Phrase tritt Schiller als Autor zurück, der in anderen Gattungen Werke produziert, also als Dramatiker, Lyriker, Historiker, Philosoph. Die Phrase - oder die Sentenz - ist sein wichtigstes Genre, da ist er am produktivsten. Das muss keine Kritik sein. Die Sentenz gilt als eine in sich geschlossene Aussage im Rahmen eines anderen Textes, die in diesem Text „das Konkret-Einmalige“ einer besonderen Situation transparent für „die Wahrheit der Idee“ machen soll. ${ }^{34}$ Solche Sätze sind zwar mit der Handlung verbunden, erreichen aber auch wegen der Abgesetztheit der Formulierungen eine „relative Einzelexistenz“. ${ }^{35}$ So gesehen konstituiert die Sentenz ein Genre, das nur innerhalb eines anderen Genres vorkommt. ${ }^{36}$

27 Gerhard (1998), S. 759 und 763

28 Michael Hofmann (2005), S. $561-562$.

29 Helmstetter (2010), S. 94.

30 Oellers (1967), S. 309.

31 Siehe dazu Henry Hitchings: „Seneca said“, in: Times Literary Supplement 14.10.2011, S. 25.

32 Oellers (1967) S. 14.

33 Goetz (2008), S. 140.

34 Ueding (1971), S. 184.

35 Niemeyer (1934), S. 12

36 Geoffrey Bennington bietet eine dekonstruktive Lektüre von Sentenzen im Roman, die die Abgesetztheit der sentenzhaften Sätze problematisiert: „The sententious statements do not stand outside narrative propositions, 
Das schon früh vollzogene Herausbrechen von Sentenzen erklärt laut einigen Germanisten teilweise auch die Verwendung Schillers durch die Nationalsozialisten: „Man brauchte Schillers Gesamthaltung ja gar nicht zu verstehen“, schreibt Gero von Wilpert, „um zugkräftige Worte herauszuklauben, die wegen ihrer sprachlichen Prägnanz überzeugen sollten“. ${ }^{37}$ Schiller war genaugenommen immer für die meisten politischen Richtungen brauchbar, so Volker Dörr: „Bemerkenswert ist [...], dass Schiller von Vertretern aller politischen Richtungen und von völlig verschiedenen politischen Positionen aus funktionalisiert wird. Für dieses Verfahren attraktiv sind die Schillerschen Texte offenbar vor allem wegen der pathetischen Prägnanz der Formulierungen. “38

\section{Geflügelte Worte}

Bei der Lektüre der überlappenden Kommentare zu Schiller wird man langsam neugierig auf das, was als Phrase, Sentenz und Stammbuchstückchen benannt wird. Was zeichnet Phrasen als solche aus, wie sind Sprüche gebaut, woran lässt sich Prägnanz erkennen? Taugen die Bezeichnungen als Kategorien von Sätzen oder Ausdrücken mit gemeinsamen Merkmalen? Die Interventionen von Adorno, Brentano, Fontane, Goetz und Kraus, aber auch die germanistischen Kommentare bieten kaum Beispiele an, nicht einmal ein „Die Axt im Haus erspart den Zimmermann“.

Beispiele lassen sich aber leicht nachschlagen; es gibt sogar eine Reihe von Büchern, die Schillers beste, prägnanteste Sätze zusammentragen, aufbewahren und zur Verfügung stellen. Wie Ute Gerhard zeigt, erscheinen bereits in den ersten Jahren nach Schillers Tod Sammlungen wie Schiller's Aphorismen, Sentenzen und Maximen, über Natur, Kunst, Welt und Menschen (1806) oder Schillers Kraftsprüche für Deutsche auf die jetzigen Zeitumstände passend (1814). ${ }^{39}$ In diesen Büchern vollzieht oder verstärkt sich die konstatierte Fragmentierung von Schillers Werken: Die Sentenzen, die in den Dramen Situationen zusammenfassen und klären, werden als besonders wertvolle Highlights aus dem Gefüge der einzelnen Werke herausgenommen und als Maximen, allgemeingültige Lebensregeln, präsentiert. ${ }^{40}$

Die oben genannten Sammlungen machen deutlich, dass der Kontakt mit Schiller sehr schnell nicht nur und vielleicht auch nicht hauptsächlich über vollständige Texte oder Aufführungen seiner Dramen verläuft, sondern eher über das Weiterreichen von besonders kräftigen und klugen Sätzen. Gleichzeitig halten gerade diese Sammlungen die Verehrung Schillers aufrecht. An Schiller wird als zu ehrender Urheber erinnert; seine prägnanten Sätze sollen sich so schnell nicht in anonyme Sprichwörter verwandeln. ${ }^{41}$

Die Anthologisierung zitierbarer Sätze hat also früh angefangen und setzt sich bis heute fort, mit Büchern wie Kleines Lexikon der Schiller-Zitate und Des Lebens wechselvolles Spiel. Weisheiten. ${ }^{42}$ Die Sammlung, die alle anderen an Bedeutung überragt, sind

\footnotetext{
and simply intervene from a position of structural exteriority to comment on, correct, or draw descriptive generalizations from 'individual' events. Rather, sententiousness tends to make general the narrative itself". Allerdings leugnet Bennington nicht, dass gerade Sentenzen durch Zitierbarkeit ausgezeichnet sind: ,they [Sentenzen] can quite happily be read in isolation from their surroundings." Bennington (2007), S. 110 und S. 8.

37 von Wilpert (2009), S. 155.

38 Dörr (2005), S. 131.

39 Siehe Gerhard (1998), S. $760-761$.

40 Maximen sind ethische Grundsätze oder allgemeingültige Lebensregeln, so die Definition im Metzler Lexikon Literatur, S. 481.

${ }^{41}$ Für den größten Teil der Sprichwörter ist die Herkunft nicht bekannt. Siehe Röhrich/Mieder (1977), S. 4.

42 Prossliner (2004).
} 
aber Georg Büchmanns Geflügelte Worte, der „klassische Zitatenschatz“, ${ }^{43}$ in dem Schiller als der meistzitierte deutsche Autor auftritt. In der ersten Ausgabe von 1864 liegt er mit 24 Seiten Zitaten „deutlich vor Goethe mit 15 Seiten“, dem zweitwichtigsten Zitatproduzenten der deutschen Sprache. ${ }^{44}$ (Als ,ergiebigster Lieferant“" in der britischen Literaturgeschichte gilt Oscar Wilde, zumindest nach dem neuesten Oxford-Zitatenlexikon. ${ }^{45}$ ) Kein Buch hat im deutschen Sprachraum so lange zitierbare Sprüche bereitgestellt wie Büchmanns Anthologie, und deren Auswahl zufolge hat kein Autor so viele zitierbare Sätze geschrieben wie Schiller.

Hier findet man Zitate wie z. B. „Der Krieg ernährt den Krieg“, ${ }^{46}$ „Was ist der langen Rede kurzer Sinn?“, ${ }^{47}$ „Denn aus Gemeinem ist der Mensch gemacht, Und die Gewohnheit nennt er seine Amme“, 48 „Eng ist die Welt, und das Gehirn ist weit" , ${ }^{9}$ „Leicht beieinander wohnen die Gedanken, Doch hart im Raume stoßen sich die Sachen“, 50 „Daran erkenn ich meine Pappenheimer", 51 „Das ist das Los des Schönen auf der Erde“, 52 „Man soll den Tag nicht vor dem Abend loben", 53 alle aus Schillers Wallenstein. Aber auch bei Büchmann findet sich keine sprachliche Analyse der Merkmale von Schillers Phrasen oder prägnanten Formulierungen, denn die Pointe des Buches besteht darin, solche Begriffe mit Hilfe der im Titel angekündigten Kategorie zu ersetzen. Alle im Buch gesammelten Zitate sind eben nichts anderes als ,geflügelte Worte“, d.h. ,allgemein bekannt gewordene“ Sprüche, die aber von „nachweisbaren Verfassern“ ausgegangen sind. ${ }^{54} \mathrm{Un}$ abhängig davon, wie die sprachliche Struktur der Zitate genau zu beschreiben ist, werden sie dank ihrer besonderen „Flugfähigkeit“ aufgenommen. Entscheidend bei Büchmann ist die Beobachtung einer Beweglichkeit oder Landläufigkeit, keine Bestimmung von gemeinsamen sprachlichen Eigenschaften.

Und die offensichtliche Zirkulation der Schillerzitate wurde weiter dank der großen Popularität des Zitatenschatzes gestärkt. Schillers Verse, schreibt der Literaturwissenschaftler Hart Nibbrig, sind ,auf Büchmanns Flügeln davongeflattert" ${ }^{55}$ Darüber hinaus ließe sich behaupten, dass gerade die Aufnahme in den Zitatenschatz schon einen minimalen Beweis für die beobachtete und benannte Beweglichkeit konstituiert, dass also bereits das Zitieren einzelner Sprüche die Aufmerksamkeit auf deren besondere Zitierbarkeit lenkt. Die Kategorisierung der Sprüche als „geflügelte Worte“ durch das Buch wäre sogar durch eben dieses Auftauchen in der Anthologie gerechtfertigt. Die Anthologie würde so selber das Merkmal verleihen, das sie nur zu konstatieren scheint. ${ }^{56}$

43 Büchmann (1993).

44 Dörr (2005), S. 129.

45 edo.: „Geschmackssache. Warum wird Oscar Wilde dauernd zitiert? Das nervt!“, in: Frankfurter Allgemeine Zeitung, 17.10.2013, S. 25.

46 Büchmann (1993), S. 152.

47 Ebd., S. 153.

48 Ebd.

49 Ebd., S. 154.

50 Ebd.

51 Ebd.

52 Ebd., S. 155.

53 Ebd.

54 Georg Büchmann, zitiert in Zimmermann (2007), S. 477.

55 Hart Nibbrig (1995), S. 159.

56 Zum Problem der Auswahlkriterien oder des „Herausgeber-Dafürhaltens“ in Büchmanns Geflügelten Worten siehe Zimmermann (2007), S. 480 - 481 und 494. 
Geflügelte Worte von Büchmann erfasst geflügelte Worte, erleichtert den schnellen Zugang zu Schillers schlagfertigsten, prägnantesten Sätzen, seinen „Hammerzeilen“57, stellt eine handhabbare Best-of-Liste zusammen und befördert auf diese Weise ihren weiteren Umlauf. Im Vergleich zu anderen Autoren beruht gerade Schillers Popularität, seine Unsterblichkeit, auf seiner Zitierbarkeit, die vor allem in Büchmann, Kraftspruchsammlungen, Zitatlexika usw. evident gemacht wird. „Schiller ,lebt" und ,stirbt““ im Zitat. ${ }^{58}$ Seine Prägnanz und Zitierbarkeit beruhen, das sollte betont werden, nicht notwendig auf einer aphoristischen Schreibweise. Unter den 88 Autoren, deren Aphorismen in das 2012 publizierte Reclamheft Deutsche Aphorismen aufgenommen sind, trifft man nicht auf Schiller. ${ }^{59}$ (Der deutsche Nationaldichter Schiller hat die meisten geflügelten Worte geprägt, aber anscheinend keinen einzigen Aphorismus; vielleicht sind pointierte Aphorismen nicht nationsgründend.)

Wie Schiller aber zum Teil dank Büchmann weiterzirkuliert, das lässt sich nicht eindeutig von der Anordnung der Zitate in der Anthologie ablesen. Wie wird der fragmentierte (aber eben nicht anonymisierte) Schiller gebraucht?

\section{Organisierte Geselligkeit}

Büchmann und andere Sammlungen stellen Schiller-Zitate bereit und bestätigen und beschleunigen so die Auflösung der einzelnen Werke zugunsten der Verstärkung des Ruhms. Wie leben oder lebten die Zitate dann weiter? Um diese Frage skizzenhaft zu beantworten, können wir jetzt zurückkehren zu Kraus, Adorno und Goetz, also zu den Texten, die arm an Definitionen und Beispielen sind, dafür aber die soziale Dimension der Schillerverehrung und den Schillergebrauch in den Blick nehmen, eben weil dieser Gebrauch, das soziale Leben von Zitaten, das eigentliche Objekt ihres Interesses darstellt. Bei den eingangs zitierten Autoren findet sich eine Art Katalog der Orte und Anlässe, Rituale und Szenen, Typen und Verhalten, die Schillers Unsterblichkeit unterstützen oder eigentlich ausmachen. Die Phrase ist ein ,sozialer Vorgang“.60

Fangen wir bei Kraus an. Er spricht von Phrasen, die von Schiller stammen, sammelt sie aber nicht. Im Mittelpunkt steht stattdessen, wer Schillers geflügelte Worte wo und bei welchen Anlässen zitiert. Und Kraus' schlichte Antwort lautet: Vereinsmitglieder. Das bedeutet für ihn, ,[a]lle, die sich ihrer Persönlichkeit erst bewußt werden, wenn sie die Menschheit ans Herz drücken, und die vor dem Sturz ins Chaos sich bewahren, indem sie einen Verein gründen. Pastoren, Demokraten, Schlaraffen, Mitglieder des Vereins ,Flamme‘, Mitglieder des Vereins ,Glocke‘, überhaupt Mitglieder.“61 Das zentrale Milieu des Schillerzitierens ist, nach Kraus, die „organisierte Geselligkeit“" der bürgerlichen Schicht. ${ }^{62}$ Wenn er über die Schillerverehrung als die virulenteste Form des Dichterfeierns im deutschsprachigen Raum schreibt, thematisiert er die bürgerliche Weise, zusammenzukommen, sich gesellig zu verhalten, soziale Stabilität und Exklusivität mit Hilfe

57 Robert Gernhardt hat die Bezeichnung geprägt und Hammerzeilen als Formulierungen definiert, die sich dem „Publikum eingehämmert haben“. Gernhardt (2010), S. 38.

58 Helmstetter (2010), S. 95.

59 Spicker (2012).

60 Den Ausdruck übernehme ich aus Sigmund Freuds Der Witz und seine Beziehung zum Unbewussten (1992), S. 153. Ein Kapitel der Studie heißt „Der Witz als sozialer Vorgang.“

${ }_{61}$ Ebd., S. 28.

62 Siehe Nathaus (2009). 
der Vereinsform zu strukturieren und sich über gemeinsame (idealistische!) Werte auf feierliche Weise zu verständigen.

Im Verein als eine eminent bürgerliche Form von Sozialisierung versammeln sich Männer, oder Mitglieder, zur Schillerverehrung. Und einmal versammelt, zelebrieren sie sich vor allem selber, denn die „Lebensblindheit, die den Blick, gen Himmel“ richtet“, fühlt sich an Schillers „Ehrentag geschmeichelt““ ${ }^{63}$ Das Feiern von großen Idealen, die Schillers Phrasen evozieren, stellt vor allem ein Selbstfeiern der selbsternannten Idealisten dar. Wer Schillerzitate effektvoll anzuwenden weiß, hat an der Achtung, die Schiller genießt, persönlich teil. ${ }^{64}$

Wenn Kraus über Schillers „Unsterblichkeit“ spricht, weist er auf die soziale Form des Vereins als Dynamo der Selbst-Vergewisserung und Selbst-Begeisterung ihrer bürgerlichen Mitglieder hin. Dieses Porträt besitzt eine gewisse geschichtliche Legitimität. Rainer Noltenius' Untersuchung der öffentlichen Festkultur im 19. Jahrhundert weist darauf hin, dass die in Deutschland bedeutenden Schillerfeiern von sozial und politisch aufstrebenden Bürgern getragen wurden. Auch einige Handwerker und Arbeiter haben Schiller gefeiert, im Gegensatz zu Adligen oder Bauern; die Feste wurden jedoch von bürgerlichen „Universitätsprofessoren, Lehrern, Schriftstellern, Pastoren, Künstlern, Medizinern, Juristen, Studenten, Buchhändlern“ dominiert. ${ }^{65}$ Und sie haben Schiller nicht irgendwie vereinzelt oder ausschließlich im privaten Raum verehrt, sondern sind in die städtische Öffentlichkeit als Vereine getreten. Mit Bezug auf die vielen Schillerfeste im Jahr 1859 schreibt Noltenius: „In der Reihenfolge der Häufigkeit waren unter den allgemeinen Vereinen folgende Veranstalter [bei den Schillerfesten] aktiv: literarische Gesellschaften, Gesangvereine, Logen, religiöse Gemeinschaften, Kunstvereine und Turnvereine; bei den Berufsverbänden: Künstlervereine, Schriftstellervereine, Handwerker- und Arbeitervereine, Buchhändlerkreise, Lehrervereine, akademische und kaufmännische Vereine“".66

Noltenius' Listen von Berufen und Vereinen ergänzen die von Kraus, aber der Literaturhistoriker stellt einen weiteren Katalog zusammen, nämlich ein Verzeichnis von Orten und festlichen Ritualen, an denen und durch die Schiller gefeiert wurde. Es gab z.B. „Schillerdenkmal-Enthüllungen vor den Rathäusern, mit Festaufführungen, Prologen, Liedern, lebenden Bildern, Reden und Büstenbekränzungen in Rathäusern, Theatersälen, Schulen, Universitäten, in Gastwirtschaften, bei Festbanketten“ oder ein „städtisches Gesamtprogramm mit Festumzug, Festaufführungen, Konzerten, Festessen und Denkmalsenthüllungen“" ${ }^{67}$ Kraus beobachtet auch die Rituale des Schillerfeierns, fasst aber die wiederkehrenden Veranstaltungen in kurzen Fragen zusammen: „Warum rückt denn diese ganze freiwillige Feuerwehr aus, wenn Schiller Geburtstag hat? [...] Was bestimmt die Turnvereine, uns den Ausblick auf Schiller zu verstellen?"“68

Es sind laut Kraus das Vereinswesen und seine penetrante Festkultur, die es fast unmöglich machen, zum Gehalt von Schillers Werken vorzudringen. Er empfiehlt auch nicht, dass man wieder Schillers Texte konzentriert liest oder seine Dramen unverkürzt aufführt; so einfach ist es nicht, den Weg zur seitens der Germanistik geforderten ,pro-

\footnotetext{
63 Ebd., S. 23

64 Siehe dazu Zimmermann (2007), S. 479

65 Noltenius (1988), S. 239

66 Ebd., S. 240.

67 Ebd., S. 239. Noltenius beschreibt auch, wie das Bürgertum an kirchliche und feudale Umzugstraditionen anknüpfte. Feierliche Festzüge wurden z. B. als Wallfahrten bezeichnet, Schiller als Prophet dargestellt. Ebd., S.

68 Kraus (1909), S. 25.
} 249. 
duktiven Rezeption“ zu finden. Was Kraus zumindest halbwegs ernsthaft vorschlägt, ist ein Verhindern sozialer Zusammenkünfte, also die Auflösung der organisierten Geselligkeit, die um Schiller herum stattfindet: „Ehe wir von dem Künstler [Schiller] reden wollen, muß unbedingt auch nur die entfernteste Möglichkeit beseitigt sein, daß um eine Schillerbüste ein Männergesangverein Aufstellung nimmt. “69

Nach Kraus' Porträt der einsatzbereiten Gesangvereine und Feuerwehren steht ,Schiller" für ein Ensemble von beruflich definierten Bürgern, vereinsförmigen Kreisen, öffentlichen Orten und feierlichen Veranstaltungen..$^{70}$ Bei Festumzügen, Büstenbekränzungen und Denkmalenthüllungen kommen beruflich definierte Bürger räumlich zusammen, sehen Bekannte und Kollegen und werden gesehen, fokussieren ihre Aufmerksamkeit auf ein gemeinsames, ernsthaftes Thema, werden affiziert von den Emotionen anderer und zeigen wiederum ihre eigenen Emotionen und erzeugen so gemeinsam ein Erlebnis. ${ }^{71}$ Ein Fest findet statt, definiert als eine sozial integrative, entlastende Suspension des Alltags. ${ }^{72}$ Die ,in Gang gesetzte Zirkulation der Betörung und Begeisterung“, so Rudolf Helmstetter, ,entfaltet eine ansteckende Wirkung“ und wird dadurch „selbstläufig und selbstverstärkend, sofern sie nur Anschlüsse an und Zuflüsse von anderen Energien findet ${ }^{\text {“ }}{ }^{73}$ Durch einen solchen Prozess, den Helmstetter vielleicht etwas gesucht mit einer Häufung von Metaphern darstellt (Zirkulation, Ansteckung, Energieflüsse), entsteht ein verbreitetes, emotional intensives Gefühl der Kollektivität, aus der die Teilnehmer Selbstvergewisserung ziehen.

\section{Festlichkeit}

Es sind also feierliche und festliche Veranstaltungen, bei denen sich die bürgerlichen Vereinsmitglieder immer wieder fragen, ,wie sagt doch Schiller...“ Die Phrase und der Spruch, so könnte man Kraus' implizite These formulieren, gehören zur gehobenen Sprache des Vereins bei besonderen geselligen Gelegenheiten. 1955 spricht Ernst Bloch rückblickend von den „unzähligen Festreden“, die mit „Sentenzen“ aus Schillers Texten versehen worden sind; sein Werk scheint „wie geschaffen für tönende Anlässe“ zu sein. ${ }^{74}$ Laut Helmstetter ist Schillers Diktion auch von Anfang an von einer „festrhetorischen Tradition“"75 geprägt und Gert Ueding bezeichnet den jungen Schiller als „Meister der epideiktischen Beredsamkeit" ${ }^{76}$ Bei dem Germanisten Paul Niemeyer findet man schließlich einen Versuch, die „Festlichkeit“ als wiederkehrendes Merkmal der SchillerSentenzen aufzustellen, also Festlichkeit als rhetorische Kategorie einzuführen. ${ }^{77}$ Besonders festlich sind ihm zufolge z. B. Substantivierungen (,Der ist ein Rasender“) ${ }^{78}$ Appo-

69 Ebd., S. 24.

70 Von der politisch-nationalen Bedeutung Schillers als deutscher „Erinnerungsort“" siehe Dann (2009).

71 Siehe dazu Collins (2004), S. $33-35$.

72 Bekannt ist Odo Marquards (1994) Definition des Festes als „Moratorium des Alltags“. Josef Kopperschmidt (1999, S. 152 - 153) spricht von den kompensatorischen und integrativen Funktionen der Feste: Sie erlauben ein befristetes Aussetzen von den Forderungen des Alltags sowie eine Reinszenierung der Kollektivitität.

73 Helmstetter (2010), S. 91.

74 Bloch (1969), S. 101.

75 Helmstetter (2010), S. 107.

76 Ueding (1971). Ueding erinnert ferner daran, dass Adam Müller Schiller als den, größte Redner der deutschen Nation“ bezeichnet, der „die dichterische Form nur wählte, weil er gehört werden wollte“. Müller zitiert in Ueding (1971), S. 4.

77 Niemeyer (1934), S. 143.

78 Ebd., S. 146. 
sitionen („Der Mensch ist, der lebendig fühlende“) ${ }^{79}$ der vorangestellte Genitiv („Des Menschen Engel ist die Zeit“) ${ }^{80}$ die Voranstellung des Objekts (,Den Menschen macht sein Wille groß und klein“") $)^{81}$ usw.

Schiller wird also nicht nur mit kanonischen, von ihm selber erläuterten Begriffen wie „das Erhabene“ oder „das Pathetische“ in Verbindung gebracht, ${ }^{82}$ sondern auch mit „Festlichkeit“. Klar ist aber, dass Schiller-Zitate immer dann auftauchen, wenn Feste stattfinden:

,Durch diese hohle Gasse muss er kommen': Der Fluch des über Generationen erfolgreichen Zitats ist, dass es mit seinem Talmiglanz den Text verdunkelt, dem es entstammt. Es kann nicht anders in ihn zurückkehren denn als alternder Allerwelts-Promi, dem man schon von ferne die unzähligen Gastspiele ansieht, die er in der Gesellschaft dröhnender Festredner und schenkelklopfender Parodisten zu absolvieren hatte. Wie romantische unheimliche Doppelgänger, die sich nicht abschütteln lassen, sind die Zitatpromis beim Klassiker Schiller allgegenwärtig. ${ }^{83}$

Kraus und anderen zufolge müsste diese Glosse über das Promi-Zitat aus der Süddeutschen Zeitung im Schillerjahr 2005 auf eine gewisse Weise buchstäblich gelesen werden. Wenn Schillerstücke aufgeführt werden, kehren die geflügelten Worte tatsächlich von Festen, Banketten und Konzerten zurück, denn dort werden Zitate in der „öffentlichen Rede und im kultivierten Gespräch“ immer wieder eingesetzt. ${ }^{84}$ Dort begegnet man ihnen zuerst oder zumindest am häufigsten, dort werden sie bestimmten Situationen zugeordnet. Und die erstaunlichen Karrieren von Schillersprüchen lassen sich später, z. B. bei Aufführungen, wohl kaum ignorieren. ${ }^{85}$ Jedes geflügelte Wort ist ein peinlich gewordener Promi, der an allzu vielen Premieren und Partys teilgenommen hat. (Um mit Goetz zu reden: Jede Phrase ist ein Mini-Brandauer.)

Büchmanns Geflügelte Worte wurde als „Repertoire ${ }^{\text {"86 }}$ von Sentenzen besonders dann gebraucht, wenn eine Festrede geschrieben werden musste. ${ }^{87}$ Mit Adorno ließe sich auch sagen: Die deutsche Phrase wurde von Schiller geprägt, aber ,,am Stammtisch eingeübt“, d. h. erst dort, am Stammtisch oder in anderen halböffentlichen oder öffentlichen Räumen, in der geselligen Runde, im Verein, bei gesellig-feierlichen Anlässen, haben Bürger gelernt, wie und wozu man in verschiedenen Situationen Schillerzitate benutzen kann. Am Stammtisch lösen sie Schmunzeln und Kopfnicken aus, werden mit Freude wiedererkannt, nochmals wiederholt und ab und zu spaßhaft modifiziert. ${ }^{88}$ In den vergangenen 150 Jahren, schreibt ein anderer Feuilletonist der Süddeutschen Zeitung, konnte man ,darauf wetten“, dass jeder Spätankömmling mit dem „unverwüstlichen Schiller-Zitat“ „Spät kommt ihr - Doch Ihr kommt“ aus Wallenstein herzhaft begrüßt wurde ${ }^{89}$ Für jede

79 Ebd., S. 147.

80 Ebd., S. 148.

81 Ebd.

${ }^{82}$ Siehe z. B. die Analyse des „Pathetisch-Erhabenen im Werk Friedrich Schillers“ in Dachselt (2003), S. 203.

83 Lothar Müller: „Durch diese hohle Gasse muss er kommen“, Süddeutsche Zeitung 09.05.2005, S. 16.

84 Zimmermann (2007), S. 479.

85 Schon 1932 beschreibt Ernst Bloch die Schwierigkeit, Schiller auf der Bühne zu sprechen. Bloch (1969), S. $53-58$.

86 Zimmermann (2007), S. 480.

87 Henry Hitchings beschreibt den Gebrauch von Zitatensammlungen so: „If you have ever had to make a speech at a wedding reception, you have probably turned to a dictionary of quotations“, Hitchings (2011).

${ }_{88}$ Zur Tradition der (oft liebevollen) parodistischen Rezeption Schillers siehe Mieder (2000).

89 Ijoma Mangold: „Spät kommt ihr - Doch Ihr kommt!“, in: Süddeutsche Zeitung 09.05.2005, S. 16. 
gesellige Situation, „ob im Salon oder beim Kolonialwarenhändler“ ${ }^{90}{ }^{9}$ war ein passendes geflügeltes Wort auszusprechen, als soziale Handlung.

Im Mittelpunkt der kritischen Berichte steht, dass die Gruppenmitglieder Schiller zum Anlass nehmen, ihre Mitgliedschaft bei Festen kollektiv zu feiern. In diesem Rahmen sind die auswendig gelernten Schiller-Phrasen als zuverlässige Vehikel der sozial erzeugten Heiterkeit vielleicht deswegen so wichtig, weil sie als festgefügte, reproduzierbare Wortgruppen transsituative Permanenz besitzen und so zeitlich und räumlich verstreute Szenen miteinander verbinden und feierliche Stimmungen als feierliche Stimmungen erkennbar werden lassen. ${ }^{91}$ Die aus den textuellen Zusammenhängen losgelösten Phrasen oder Sentenzen könnten in einem sozialen Vakuum überschäumende Gefühle wohl kaum auslösen, aber sie werden von bürgerlichen Schillerfans eng an soziale Situationen und festliche Veranstaltungen geknüpft und fangen so an, als Erinnerungsstücke und Erkennungszeichen für die gemeinsame soziale Tätigkeit zu dienen. Sie sind Fetische der festlichen Geselligkeit. ${ }^{92}$ Als solche dienen sie als unentbehrliche Bestandteile und Hilfsmittel in den immer wieder in Gang gesetzten Ritualen.

\section{Gesellige Sprache}

Begeisterung wird durch gegenseitiges Affizieren sozial hergestellt; die Vereine oder eher lockere Runden feiern sich selber als Kollektive, unter Rekurs auf Schiller. In diesem Kontext stellen die festlichen Schiller-Zitate vitale Instrumente des Sich-SelbstFeierns dar. Warum ist es (oder war es) aber so schön, solche geflügelten Worte auszusprechen, gerade im geselligen Kreise? Woher kommt der gemeinsame Genuss?

Passende geflügelte Worte können gewiss als Bildungsnachweise, Zugehörigkeitsformeln oder effiziente Idealismusbestätigungen dienen. Beim wiederholten Ausspruch eingeprägter Wortgruppen geht es vielleicht aber rein kognitiv um genussvolle Aufwandsersparnis. Auswendig gelernte Schillersprüche spricht man mühelos, ohne neue Leistung, und befriedigt dadurch die ,inclination toward psychic parsimony“, die den seelischen Apparat auszeichnet. ${ }^{93}$ Geflügelte Worte, schreibt Burkhard Müller, sind in erster Linie Gewohnheiten und in ihnen steckt, ,wie in jeder Gewohnheit, viel entlastende Faulheit." ${ }^{\prime 94}$ Der Genuss könnte dann in nichts anderem als eben in Aufwandsersparnissen bestehen; kognitive Entlastung fühlt sich gut an. ${ }^{95}$ Aber das Nachsprechen ist zugleich ein Sprechen mit geringem Aufwand, das zumindest bei Freunden oder im Verein gerade nicht als manifeste Faulheit gilt, sondern als legitime Kommunikation, und zuverlässig mit Anerkennung belohnt wird. Das Zitieren vertrauter, tief eingeprägter Phrasen kommt der haushälterischen Tendenz des seelischen Apparats entgegen, aber auf eine sozial zelebrierte Weise.

90 Carbe (2005), S. 62.

91 Über die Entstehung und Funktion kollektiver Symbole schreibt Randall Collins (2004, S. 37): „In actuality, the group is focusing on its own feeling of intersubjectivity, its own shared emotion; but it has no way of representing this fleeting feeling, except by representing it as embodied in an object. It reifies its experience, makes it thing-like, and thus an emblem".

${ }_{92}$ Zum Fetischbegriff siehe Wayne (2003)

93 Gray (2013), S. 101.

94 Müller (2003), S. 1095

95 Sigmund Freud meint, dass der „Lustgewinn“ bei einem erzählten tendenziösen Witz dem ersparten psychischen Hemmungs- oder Unterdrückungsaufwand entspricht. Sigmund Freud (1992), S. 132. Odo Marquard behauptet auf ähnliche Weise, dass ,,die Erleichterung, die aus seiner Bornierungsersparung resultiert“ einen entsprechenden „Glücksertrag“ ergibt. Marquard (1995), S. 35. 
Hier kann hinzugefügt werden, dass auch diejenigen, die in der sozialen Situation momentan nur zuhören, dennoch die durch Wiederholung eingravierten Zeilen durch subvokales Aussprechen begleiten, denn ohne uns dessen bewusst zu sein, simulieren wir beim Lauschen das Sprechen anderer. Je bekannter die Redewendung, desto einfacher und reibungsloser wird dieses Mitsprechen ausgelöst. ${ }^{96}$ Unter Schillerverehrern wird Schiller also immer kollektiv zitiert. Auch in diesem Sinne ist das geflügelte Wort ein sozialer Vorgang: Man spricht es nicht alleine aus.

Die geselligen Bürger, die Schillersprüche parat haben, bilden eine nach-klassische ${ }^{97}$ Allianz der Faulen, denn die Mitglieder erlauben und ermuntern einander gegenseitig, zu sprechen, ohne selber zu sprechen, und genießen die daraus resultierenden Energieersparnisse als geteilte Heiterkeit. Wo das geflügelte Wort ,gesprochen, gehört, verstanden wird“, findet, wie beim Erzählen von Witzen, ,ein kleines Fest statt“, so Burkhard Müller. ${ }^{98}$ Bei jedem geläufigen Schillerzitat sind wir in gewisser Weise in einer kleineren oder größeren Feier begriffen. Nur außenstehende Beobachter, Figuren wie Kraus, prangern das festliche Schiller-Zitieren als kollektiv organisierte Bequemlichkeit an, als applaudierte Faulheit. Vielleicht lautet die grundsätzliche Frage der Phrasenkritik: Wann und wie dürfen wir feiern?

\section{Applaus}

Goetz nennt Schiller den Mann der Phrase und liefert einen kurzen Rückblick auf den langen Weg der Schiller-Sprüche durch die (Fest-)Kultur des 19. Jahrhunderts bis zum Nationalsozialismus. Er spricht von dem „Triumphzug“ der Kalauer und Lebensweisheiten durch „die Spießerkultur“ ins „hochaggressive Hochspießertum“, von dem Schiller „ultimativ abgefeiert“ wurde. ${ }^{99}$ Auch bei Goetz geht es andeutungsweise um die Konstellation Schiller-Phrase und Schiller-Feier. Aber Goetz lebt nicht im Zeitalter bürgerlicher Geselligkeit. Den Typus, bei dem gesellige Situationen und Feste automatisch SchillerSprüche hervorrufen und der ,einen enthusiastischen Bildungsbegriff mit absoluter Unoriginalität" verbindet, gibt es nicht mehr. ${ }^{100}$ Die Tradierung der Phrase am Stammtisch ist abgebrochen. ${ }^{101}$

Das Klatschen lässt sich trotzdem nicht wegdenken, auch nach dem Niedergang der Gesangvereine nicht. Goetz behauptet emphatisch, dass die verschiedenen Formen des begeisterten Einverständnisses und Feierns bei Schiller selber schon angelegt sind. Seine Schreibweise ist so ausgerichtet, dass er von möglichst vielen geehrt werden konnte.

96 Siehe dazu Topolinski/Strack (2009). Aus dem Abstract: „The authors apply an embodied account to mere exposure, arguing that through the repeated exposure of a particular stimulus, motor responses specifically associated to that stimulus are repeatedly simulated, thus trained, and become increasingly fluent. This increased fluency drives preferences for repeated stimuli. This hypothesis was tested by blocking stimulus-specific motor simulations during repeated exposure. In Experiment 1, chewing gum while evaluating stimuli destroyed mere exposure effects (MEEs) for words“.

${ }_{97}$ Der Germanist Heinz Schlaffer schreibt: „Nach dem Ende der Revolutions- und Befreiungskriege (1815), nach dem Tod Hegels (1831) und Goethes (1832) wird die nachfolgende Generation gewahr, daß ihre intellektuellen Leistungen nicht auf der Höhe der früheren Epoche stehen. Die literarische Produktion geht weiter, aber sie kommt nicht voran." Diese nachklassische Zeit des literarischen Vegetierens ist auch die Epoche der geflügelten Worte. Schlaffer (2000), S. 115 - 116.

98 Müller (2003), S. 1089.

99 Goetz (2008), S. 140.

100 Ijoma Mangold: „Spät kommt ihr - Doch Ihr kommt!“, in: Süddeutsche Zeitung 09.05.2005, S. 16.

101 Mit Blick auf Neubearbeitungen von Büchmanns Geflügelten Worten schreibt Rolf Zimmermann (2007, S. 488), dass ,das öffentliche Gespräch von heute [...] offenbar immer weniger mit und von der Literatur“ lebt. 
Bereits die Sprüche, aus denen seine Stücke teilweise gebaut sind, zielen vor allem auf Bestätigung. Bei Schiller spürt man laut Goetz, wie die erhoffte Zustimmung -,,das stimmt, das habe ich mir auch schon oft gedacht" - jeden gelungenen sentenziösen Satz begleitet. ${ }^{102}$ Schon in dem Gefüge seiner Sprache ist so zu bemerken, wie Schiller im Weimar der 1790er Jahre seinen Erfolg auf „eine unangenehme Anhimmelkultur“ gegründet hat. ${ }^{103}$ Sentenzen wollen vom Publikum ${ }^{104}$ applaudiert werden und scheinbar allgemeingültige Sprüche sind allzu verständliche und deswegen sozial erfolgreiche Vereinfachungen; das sind gegen Schiller gerichtete Anklagen.

Mai 2007. Die zehnstündige Schilleraufführung mit Klaus Maria Brandauer sei, so die Rezensionen, ein wahrer „Schiller-Marathon“105 mit Brandauer in einer „Mammut-Rolle" gewesen. ${ }^{106}$ Und Brandauer selber versprach in einem Interview ein sommerliches „Schiller-Woodstock“. ${ }^{107}$ Goetz befindet sich, so ließe sich das vielleicht ausdrücken, in einer Kultureventkultur, mit starbesetzten Großprojekten in umgebauten Brauereien. Das extrem lange Stück ist als Festival zu verstehen und soll vor allem Eindruck machen, Größe ausstrahlen. Jubel kommt denn auch vor: „Begeisterung im Publikum: absolut, grenzenlos, ohne Widerspruch. Die Leute jubelten. Das war am Ende, nach gut 10 Stunden, der endgültige Abturn, wie die Leute rasten und sich freuten, die fanden diese Müllkippe von Theater wirklich grandios. “108 Und einige Absätze später fügt Goetz hinzu: „Vielleicht ist Applaus, du musst es nochmal sagen, ja überhaupt das Allerscheußlichste auf Erden." ${ }^{109}$

\section{Literaturverzeichnis}

Adorno, Theodor W. (2004): Gesammelte Schriften, Band 4, hg. von Rolf Tiedemann, Frankfurt am Main.

Benn, Gottfried (1954/2001): „Anthologien. Zum »Tag des Buches« am 27. November 1954“. In: Benn, Gottfried: Sämtliche Werke. Stuttgarter Ausgabe, Band. VI, Stuttgart, S. $158-161$.

Bennington, Geoffrey (2007): Sententiousness and the Novel. Laying Down the Law in Eighteenth-Century French Fiction. Cambridge UK.

Bloch, Ernst (1969): Die Kunst, Schiller zu sprechen und andere literarische Aufsätze. Frankfurt am Main.

Brentano, Clemens (1963): Werke, Band 2, hg. von Friedhelm Kemp, Darmstadt.

Büchmann, Georg (1993): Geflügelte Worte: der klassische Zitatenschatz, neu bearb. von Winfried Homann, 39. Auflage, Frankfurt am Main.

Bullinger, Matthias (2013): ,Jeden Donnerstag um Sieben“. In Schrank, Volker: Stammtische, Berlin, S. 5-9.

\footnotetext{
102 Goetz (2008), S. 140

103 Ebd., S. 140.

104 „Das Publikum“, schrieb Schiller 1784, ,ist mir jetzt alles, mein Studium, mein Souverain, mein Vertrauter. Ihm allein gehör ich jetzt an.“Zitiert in Müller (1986), S. 151.

105 Esther Slevogt, „Überwältigungsästhetik im Breitwandformat“. http://www.nachtkritik.de

106 Spiegel Online zitiert in: Esther Slevogt, „Überwältigungsästhetik im Breitwandformat“. Ebd.

107 Brandauer kündigt Schiller-Woodstock an, Der Tagesspiegel 07.05.2007 unter http://www.tagesspiegel. de/kultur/wallenstein-inszenierung-brandauer-kuendigt-schiller-woodstock-an/844056.html.

108 Goetz (2008), S. 137.

109 Ebd., S. 138. 2013 hat Rainald Goetz den Schiller-Gedächtnis-Preis erhalten. Auch in seiner Dankrede macht er kritische Bemerkungen zu Schiller. Sie sind aber milder: Schiller „hat kein Ich gehabt, keine Sprache und deshalb auch keinen Roman. Aber er hatte Ideen, Gesellschaft und die große Ambition zu WIRKEN. So konnte er seine höchst effektiven Theaterstücke so gut schreiben." Goetz (2014), S. 9.
} 
Bunia, Remigius/Dembeck, Till/Stanitzek, Georg (Hg.) (2011): Philister. Problemgeschichte einer Sozialfigur der neueren deutschen Literatur. Berlin.

Carbe, Monika (2005): Schiller. Vom Wandel eines Dichterbildes. Darmstadt.

Collins, Randall (2004): Interaction Ritual Chains. Princeton.

Dachselt, Rainer (2003): Pathos. Tradition und Aktualität einer vergessenen Kategorie der Poetik. Heidelberg.

Dann, Otto (2009): „Schiller“. In: François, Etienne/Schulze, Hagen: Deutsche Erinnerungsorte, Band II. München 2009, S. 170-186.

Darsow, Götz-Lothar (2000): Friedrich Schiller. Stuttgart.

Dörr, Volker C. (2005): Friedrich Schiller. Frankfurt am Main.

Freud, Sigmund (1992): Der Witz und seine Beziehung zum Unbewussten. Frankfurt am Main.

Gerhard, Ute (1998): „Schiller im 19. Jahrhundert“. In: Schiller-Handbuch, hg. von Helmut Koopmann, Stuttgart, S. 758- 772.

Gernhardt, Robert (2010): Was das Gedicht alles kann: Alles. Texte zur Poetik, hg. v.Lutz Hagestedt und Johannes Möller, Frankfurt am Main.

Goetz, Rainald (2008): Klage. Frankfurt am Main.

- (2014): „Schiller-Rede“, in: Zeitschrift für Ideengeschichte 8, Heft 1, S. 5-9.

Gray, Richard T. (2013): „Economics as a Laughing Matter: Freud's Jokes and Their Relation to the Economic and Rhetorical Unconscious“, in: The Germanic Review, Heft 88, S. 97-120.

Hart Nibbrig, Christian L. (1995): Ästhetik des Todes. Frankfurt am Main.

Helmstetter, Rudolf (2010): „Schillers Tode und Unsterblichkeiten“, in: Helmstetter, Rudolf/Meyer, Holt/Müller Nielaba, Daniel (Hg.): Schiller. Gedenken -Vergessen - Lesen, S. 87-122. München.

Hitchings, Henry (2011): „Seneca said“, in: Times Literary Supplement 14.10.2011, S. 25.

Hofmann, Michael (2005): „Wirkungsgeschichte“, in: Matthias Luserke-Jaqui (Hg.) „Schiller-Handbuch“, Stuttgart, S. 561-581.

Kopperschmidt, Josef (1999): „Über die Unfähigkeit zu feiern. Allgemeine und spezifisch deutsche Schwierigkeiten mit der Gedenkrhetorik“, in: Kopperschmidt, Josef/ Schanze, Helmut (Hg.): Fest und Festrhetorik. Zur Theorie, Geschichte und Praxis der Epideiktik. München, S. 149-172.

Kraus, Karl (1909): „Schrecken der Unsterblichkeit“, in: Die Fackel 11, Heft 291, S. 23-28.

Mangold, Ijoma (2005): „Spät kommt ihr - Doch Ihr kommt!“, in: Süddeutsche Zeitung 09.05.2005, S. 16 .

Mann, Michael (2004): Fascists. Cambridge UK.

Marquard, Odo (1994): „Moratorium des Alltags. Eine kleine Philosophie des Festes“, in Skepsis und Zustimmung. Stuttgart, S. 59-69.

Marquard, Odo (1995): Glück im Unglück. Philosophische Überlegungen. München.

Mieder, Wolfgang (2000): Aphorismen, Sprichwörter, Zitate von Goethe und Schiller bis Victor Klemperer. Bern.

Müller, Burkhard (2003): „Die Flügel des Gedächtnisses“, in: Merkur 57, S. 1087-1099.

Müller, Klaus-Detlef (1986): „Schiller und das Mäzenat. Zu der Entstehungsgeschichte der ,Briefe über die ästhetische Erziehung des Menschen “", in: Barner, Wilfried/Lämmert, Eberhard/Oellers, Norbert (Hg.): Unser Commercium. Goethes und Schillers Literaturpolitik. Stuttgart, S. 151-167.

Müller, Lothar (2005): „Durch diese hohle Gasse muss er kommen“, in: Süddeutsche Zeitung 09.05.2005, S. 16. 
Nathaus, Klaus (2009): Organisierte Geselligkeit. Deutsche und britische Vereine im 19. und 20. Jahrhundert “, Göttingen.

Niemeyer, Paul (1934): Die Sentenz als poetische Ausdrucksform vorzüglich im dramatischen Stil. Untersuchungen an Hand der Sentenz in Schillers Drama. Berlin.

Noltenius, Rainer (1988): „Schiller als Führer und Heiland. Das Schillerfest 1859 als nationaler Traum von der Geburt des zweiten deutschen Kaiserreichs“, in: Düding, Dieter/Friedemann, Peter/Münch, Paul (Hg.): Öffentliche Festkultur. Politische Feste in Deutschland von der Aufklärung bis zum Ersten Weltkrieg. Reinbek, S. 237- 258.

Oellers, Norbert (1967): Schiller. Geschichte seiner Wirkung bis zu Goethes Tod, $1805-$ 1832. Bonn.

Oellers, Norbert (1970): „Einleitung“, in: ders.: Schiller - Zeitgenosse aller Epochen. Dokumente zur Wirkungsgeschichte Schillers in Deutschland. Teil 1, München, S. $1-53$.

Prossliner, Johann (2004): Kleines Lexikon der Schiller-Zitate. München

Röhrich, Lutz/Mieder, Wolfgang (1977): Sprichwort. Stuttgart.

Schiller, Friedrich (2004): Des Lebens wechselvolles Spiel. Weisheiten. Hg. v. Johann Prossliner. München.

Schlaffer, Heinz (2000): Die kurze Geschichte der deutschen Literatur. München.

Slevogt, Esther: „Überwältigungsästhetik im Breitwandformat“, in: http://www.nachtkritik.de

Spicker, Friedemann (Hg.) (2012): Deutsche Aphorismen. Dietzingen.

Topolinski, Sasha/ Strack, Fritz (2009): „Motormouth. Mere exposure depends on stimulus-specific motor simulations", in: Journal of Experimental Psychology: Learning, Memory, and Cognition 35, Heft 2, S. 423-433.

Ueding, Gert (1971): Schillers Rhetorik. Idealistische Wirkungsästhetik und rhetorische Tradition. Tübingen.

Vogl, Joseph/Kluge, Alexander (2009): Soll und Haben: Fernsehgespräche. Zürich.

von Wilpert, Gero (2009): Die 101 wichtigsten Fragen. Schiller. München.

Wayne, Mark (2003): Marxism and Media Studies. Key Concepts and Contemporary Trends. London.

Wenzelburger, Dietmar/Kaiser, Christine (2007): „Phrase“, in: Metzler Lexikon Literatur, hg. von Dieter Burdorf, Christoph Fasbender, Burkhard Moennighoff, Stuttgart 2007, S. 585.

Zimmermann, Rolf Christian (2007): „Der stille Rückzug der Literaturzitate aus Büchmanns Geflügelten Worten“, in: Deutsche Vierteljahrsschrift für Literaturwissenschaft und Geistesgeschichte 81, Heft 3, S. 475-499. 